A new RF acoustic method

\title{
A new radio-frequency acoustic method for remote study of liquids
}

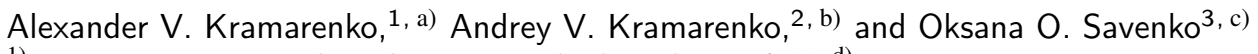 \\ ${ }^{1)}$ TREDEX Company Ltd, PO box 11515, Kharkiv, Ukraine 6100 (क) \\ ${ }^{2)}$ General and Inorganic Chemistry Department, National Technical University "KhPI”, 2 Kyrpychova Str., Kharkiv, \\ Ukraine 61002 \\ 3) School of Radiophysics, Biomedical Electronics and Computer Systems, Karazin Kharkiv National University, 4 Svobody Sq., \\ Kharkiv, Ukraine 61022
}

(Dated: 13 July 2020)

In the present work, a novel conductive liquids method of study has been proposed. It is based on the phenomenon of radiofrequency anisotropy of electrolyte solution discovered by us. It arises in response to mechanical or acoustic excitation of the solution. We have observed the phenomenon during the development of an RF polarimetric contactless cardiograph. The electric field vector of the transmitted $433.82 \mathrm{MHz}$ signal rotated after its transition through the pericardial region. That rotation depends on the change of blood acceleration when passing through the chambers of the heart and large vessels. It has also been revealed that rotation occurs after RF wave passage through the physiological saline $(0.9 \% \mathrm{NaCl})$ subjected to any mechanical excitation inside it like a jet appearing or soundwave passing. No significant difference was detected experimentally between $\mathrm{NaCl}$ and $\mathrm{KCl}$ solutions behavior. It means that the mechanism of hydrodynamic separation of ions is apparently not suitable to explain the phenomenon. The response we have registered resembles the magnetization process of spin glasses. From the nature of the observed response, we have concluded that a fundamentally new physical effect is discovered. It may provide wide opportunities for remote measurement of the electrolyte solution parameters with polarized radio-frequency signals.

Keywords: contactless cardiography, polarization vector, polarimetry, aqueous electrolyte solution, relaxation time

\section{INTRODUCTION}

Back in 1845, Michael Faraday predicted the electric field vector rotation of linearly polarized light in an applied magnetic field. And when John Kerr had discovered optical material refractive index change to be proportional to the square of the external electric field strength, the widespread use of this electro-optical phenomenon began at once. Utilizing a certain substance capable of indirect changing the polarization of electromagnetic radiation passing through was a necessary condition for Kerr, Pockels (and other) cells to work. In terms of radiophysics one may represent it like a parametric impact on the system consisting of transmitter, receiver and propagated wave. Hence the hypothesis presuming the existence of Faraday phenomenon at radio frequencies looks plausible enough.

In present work we propose a completely new conductive liquids method of study based on physical phenomenon that has never been noticed earlier. The phenomenon is the radiofrequency anisotropy of electrolyte solution excited mechanically or acoustically. The closest effect we have found in literature is the RF anisotropy discovered for solid ice ${ }^{1-5}$ or liquid crystals 6 , but not for rather unstructured aqueous solution.

The effect described in present work has unexpectedly been discovered whereas we were testing our novel device. The device demonstrates a new type of contactless cardiography which is important for assessment of human health status

\footnotetext{
a)Electronic mail: tredexcompany37@gmail.com

b)Electronic mail: kramarenko@kpi.kharkov.ua

c)Electronic mail: sombercy@gmail.com

d.http://www.tredex-company.com/en
}

when the application of ECG electrodes is impossible or inconvenient.

There is a range of papers describing the contactless ECG registration devices developed in recent years. Nowadays there is a trend of replacement of a high-impedance lowfrequency amplifying technique ${ }^{7-11}$ with an active S- or Cband radar systems 12 which are directly measure the displacement of the cardiac wall instead of electric potential recording. And they really seems to be more promising due to their better signal/noise ratio within a working band.

Our contactless polarimetric cardiography system was developed primarily for the car drivers. In this case, we had to note the interference because of vibration and displacement of the sensors must be minimized before a digital processing stage. However, up-to-date monostatic Doppler radars require either on-body antenna fixation ${ }^{12}$ or additional information about the variation of their relative position, which is several orders of magnitude greater than the amplitude of the periodic baseband signal caused by heart and breathing activity 13 . To obtain a good reflected signal (in the case of monostatic radar) the required radiation power will always be much higher than in the case of "open" operation when the locator is bistatic 17 . Minimizing of the power consumption during prolonged operation of the device is one of the most important equipment requirements 18 -20. In order to make the total RF energy flux density be lower than $25 \mu \mathrm{W} / \mathrm{cm}^{2}$ to comply ${ }^{18}$ we have used a very low power transmitter $(-6 \ldots-12 \mathrm{dBm})$. Moreover, according to our measurements, when the wave from our device passes through the human body, attenuation is small, no more than $20 \mathrm{~dB}$. It means the negligible radiation powers of $-40 \ldots-60 \mathrm{dBm}$ can be easily achieved and then the total absorbed dose of RF energy will be reduced. The signal-to-noise ratio remains good precisely because of polarimetry since an external non-polarized interference is the same in both receiv- 


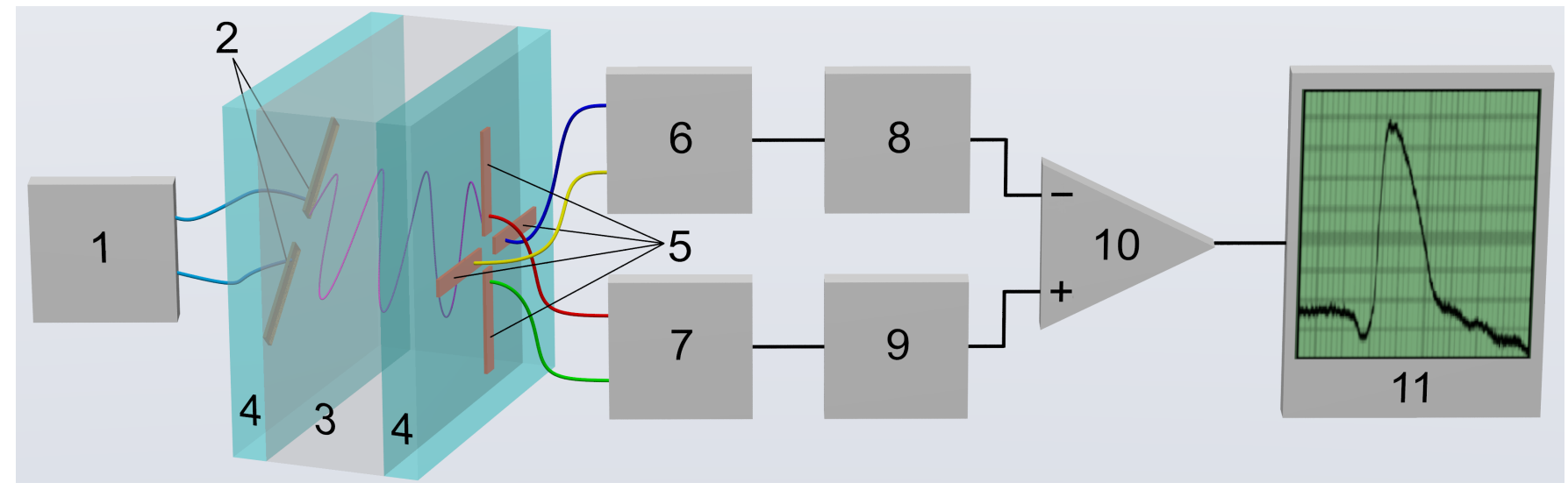

FIG. 1. A simplified block diagram of the device for recording of RF wave polarization direction deviation.

1 - a continuous wave transmitter $433.82 \mathrm{MHz},-12 \mathrm{dBm} ; 2$ - transmitting antenna; 3 - an object studied; 4 - a dielectric material; 5 orthogonal orientation quadrature antennas; 6 - channel X RF amplifier; 7 - channel Y RF amplifier; 8 - X channel amplitude logarithmic detector; 9 - Y channel amplitude logarithmic detector; 10 - instrumentation differential amplifier; 11 - registrating device (X axis is time, $\mathrm{Y}$ axis is deviation). The antennas are shown as Hertz's half-wave dipoles, it is clear that their real configuration will be different. Transmitting antenna is deviated at angle of $45^{\circ}$ relative to both receiving ones.

ing channels and is subtracted mutually.

The polarimetric method also does not impose high requirements on the spectral purity of the local oscillator signal, which is the main fundamental sensitivity limitation of continuous wave Doppler radars 21 . Therefore, the gain in minimizing the power of the emitted signal also increases many times with respect to them.

\section{METHOD BASICS}

\section{A. A brief description of the device}

Figure 1 shows a signal pathway block diagram. RF signal of the transmitter 1 excites the transmitting antenna 2. A linearly polarized wave passes through the object 3 (some abstract medium is shown here) to be received then by two orthogonally oriented antennas 5 . The input stages 6 ( $X$ channel) and 7 ( $Y$ channel) amplify the corresponding signals that pass to the amplitude logarithmic detectors 8 and 9 next. The differential amplifier 10 provides an output signal proportional to the difference in logarithms of amplitudes of lowfrequency signals. The standard $-3 \mathrm{~dB}$ bandwidth (with $0.3 \mathrm{~Hz}$ and $75 \mathrm{~Hz}$ cutoff frequencies) is applied. The dynamics of the process can be observed via the oscilloscope 11. So, the multistatic radar emitting a continuous monochromatic wave passing through the pericardial region has been implemented. Such registration principle makes our device much safer for long-term recordings (monitoring) in the sense of absorbed dose of radiation. A comprehensive paper about our system is going to be printed in the offing.

\section{B. Basic relationships between the signals}

Of course, we are far from claiming our transmitting antenna emits exactly the linearly polarized wave having no traces of elliptical polarization. But nevertheless we can reasonably assume the imaginary component of the Jones vector to be small in comparison with the real one ${ }^{22+24}$. It suggests the relation of received RF-signals to be proportional to the tangent of the shift between transmitting and receiving antenna while there is no anisotropic medium between the antennas. In order to provide this we have adjusted the antennas disposal so that the logarithmic detectors' positive output signals were out of phase relatively to certain chosen level. Considering this level as zero one we can write the final relations as:

$$
\left\{\begin{array}{l}
\log U_{y}+\log U_{x}=0 \\
\log U_{y}-\log U_{x}=\log \frac{U_{y}}{U_{x}}=\log (\tan \alpha)=U_{\text {out }} / k
\end{array}\right.
$$

where $\alpha$ is the shift, $U_{\text {out }}$ is output voltage and $k$ is the gain of op-amp 10. As the shift is $45^{\circ}$ (see fig. 1) the relation of the amplitudes is equal to one, so the relation logarithm is zero. We have also performed some gain calibration deviating $\alpha$ by $\approx \pm 30^{\circ}$ from the $45^{\circ}$ level and adjusting $U_{\text {out }}$ to be inside $\pm 1 V$ range. As a result we have derived a calibration dependence of the form of:

$$
\alpha \approx \arctan \left(\exp \left(1.32 U_{\text {out }}\right)\right) .
$$

It has turned out to be linear enough within $U_{\text {out }}$ range of $\pm 0.2 \mathrm{~V}$; the Taylor series in the vicinity of the point $U_{\text {out }}=0$ limited to the first three significant terms is

$$
\alpha \approx 45+37.8 U_{\text {out }}-11.0 U_{\text {out }}^{3} .
$$

The appearance of anisotropic medium between the antennas changes the output signal and, hence, the $\alpha$ angle variation is observed. 

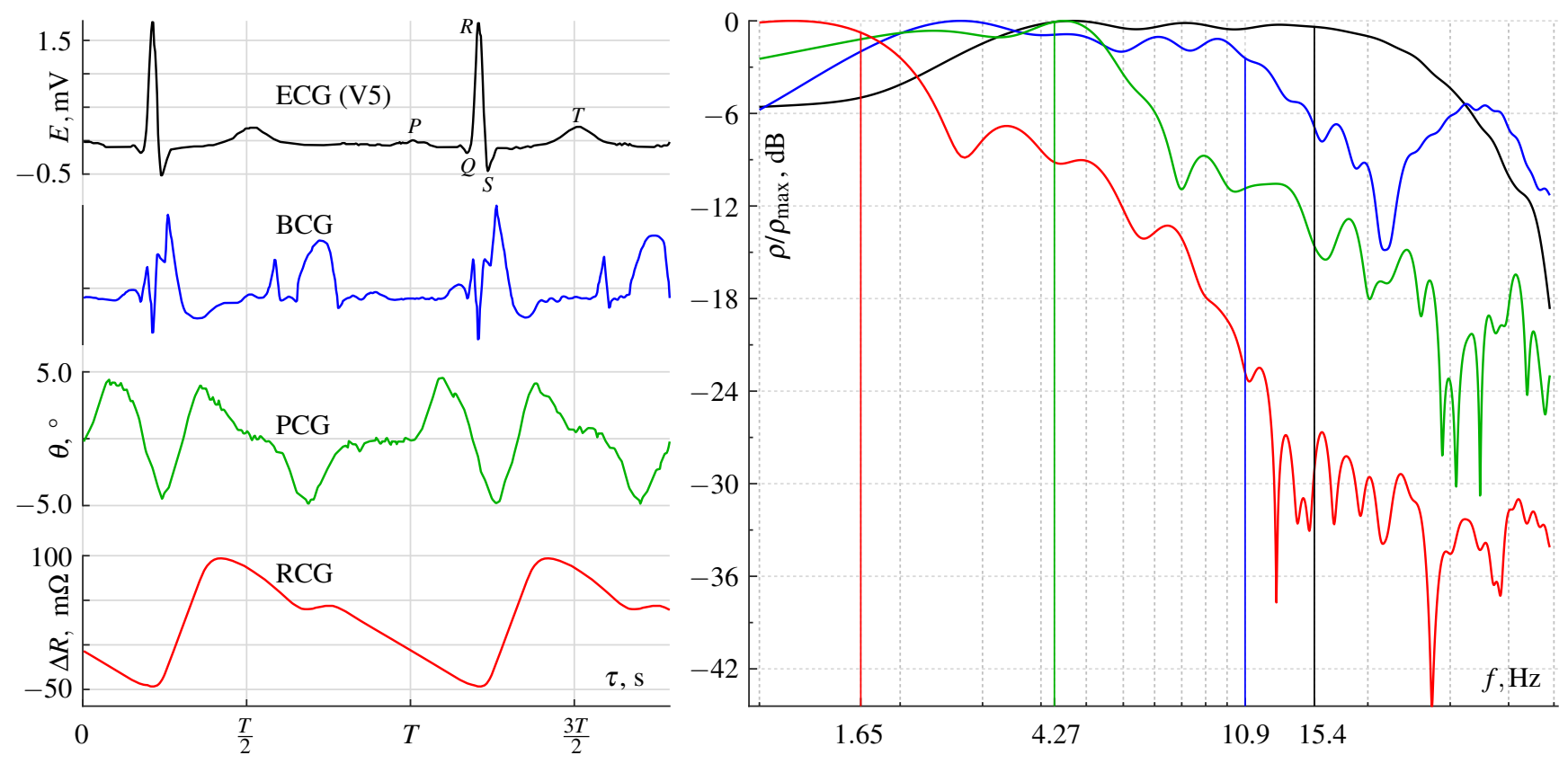

FIG. 2. The left graph shows the polarimetric cardiogram (PCG, green) we have recorded synchronously with a standard ECG in V5 lead (black) and rheocardiogram (RCG, red) combined with the ballistocardiogram 25 (BCG, blue). Right graph shows their normalized power spectra and corresponding median frequencies. The heartbeat period $T \approx 0.78 \mathrm{~s}$.

A propagation medium is commonly described ${ }^{26}$ by $2 \times 2$ scattering matrix, or operator, acting on the Jones vector of the wave. Generally such operator may change the polarization ellipticity. But we assume the pericardial region to possess only the property to rotate the polarization direction at a certain angle, making it possible to write the corresponding rotation operator $\|R(\theta)\|$ as the following ${ }^{26}$ :

$$
\begin{aligned}
& \overrightarrow{E_{0}}=\|R(\theta)\| \vec{E}= \\
& =\frac{1}{\sqrt{2}}\left\|\begin{array}{rr}
\cos \theta-\sin \theta & \cos \theta+\sin \theta\|\| E_{x} \\
-\cos \theta-\sin \theta & \cos \theta-\sin \theta \| \\
E_{y}
\end{array}\right\|,
\end{aligned}
$$

where $E_{0}$ is a Jones vector of received wave in the basis of the receiver antennas; $E$ is a Jones vector of the transmitted wave in the basis of the transmitter antenna; $\theta=\alpha-45^{\circ}$ is the angle of polarization direction rotation conditioned by the presence of anisotropic medium. All our further considerations are related to this parameter. There were no $\theta$ values more than $\pm 30^{\circ}$ recorded during testing, so all of $\alpha$ values are in the first quadrant. It allows us not to take into account the phase relation of the RF-signals received. Indeed, we are not able to distinguish polarization vector rotation and polarization ellipticity changing on the described device, observing only the output signal of the op-amp 10 .

Thus, we are able to record (with acceptable measurement error) UHF-band wave polarization change because of propagation medium anisotropy disregarding slight ellipticity of the wave. We have chosen the frequency $(433.82 \mathrm{MHz})$ so that the half-wavelength $(34.5 \mathrm{~cm})$ approximates the linear dimensions of the heart and large vessels.

\section{METHOD TESTING}

\section{A. Treatment of the data obtained and a new hypothesis}

A polarimetric cardiogram (PCG) had been expected to have minimum artifacts and was recorded by the contactless method (see the videc ${ }^{27}$ of the first successful attempt of such recording). The PCG was recorded from healthy patient who was in the middle between the receiving and transmitting antennas placed at about $1 \mathrm{~m}$ distance from each other.

The left graph in the figure 2 shows an electrocardiogram (ECG), a PCG, and a rheocardiogram (RCG) we have recorded synchronously from the same patient. We also have added another patient ballistocardiogram ${ }^{25}$ (BCG) adjusting its heartbeat period to correspond to our record. The right graph shows the corresponding power spectra of a heartbeat period. Hann window (Tukey window ${ }^{28}$ having $\alpha=1$ ) has been applied to data prior to Fourier transform.

At the beginning of polarimetric cardiography investigation we assumed the polarization direction rotation mechanism to be caused by heterogeneity of the heart as propagation medium. It is known that cardiac muscle (myocardium) and large vessels connected to it are deformed and rotated during the systole. This has been reliably sustained with dynamic studies of cardiac activity 29 . Indeed, the radio wave passing through the pericardial region changes its polarization direction because of the heterogeneous tissues shifting and rotating. It means that expected rotation of polarization direction might be synchronized with "external" cardiac ballistics.

But the PCG pulses (see figure 2) mismatch the initial hy- 
pothesis. They are more prolonged than ECG pulses, they start earlier and cease later, what defied explanation. PCG signal is in a good accordance with intracardiac (NB!) hemodynamics. It has turned out that $\theta$ change is associated rather with "internal" cardiac ballistics than with "external" one. In other words, the rotation is caused by moving of intracardiac and intravascular blood (inside the heart and large vessels connected to it).

We hypothesized that radio wave polarization direction after its passage through the pericardial region depends on the electric properties of the medium itself i.e. on the properties of the blood the radio wave penetrates. In order to prove this hypothesis we have calculated the median frequency for each the spectra we have obtained. The calculated values are shown as the $x$-axis tick labels of the right graph in the figure 2. It is well known that ECG represents the propagation process of an electric excitation wave through the myocardium. Such process might be considered as virtually inertialess and its spectrum possesses a maximum median frequency in comparison with any other cardiac monitoring methods. A ballistic cardiogram (BCG) presents the mechanical oscillations initiated by an electric signal. Since the heart contraction energy remains constant, BCG spectrum should demonstrate a red shift relatively to the ECG spectrum due to the inertia of the masses involved in oscillation process. The spectra graph (figure 2) shows it well $(10.9 \mathrm{~Hz}<15.4 \mathrm{~Hz})$ despite the high frequencies presence caused by the heart valves work ${ }^{30 \mid 31}$. If the PCG waves corresponded to the blood acceleration/deceleration and not to the myocardium contraction, the spectrum of PCG would contain far lower frequencies than the BCG spectrum. It really takes place as we can see $(4.27 \mathrm{~Hz}<10.9 \mathrm{~Hz})$ what backs our hypothesis. And, of course, the RCG spectrum should have the lowest median frequency since a rheocardiogram signal depends (according to Kedrov ${ }^{32}$ and Nyboes ${ }^{33}$ ) on a tissue volume variation affected by newly run blood. The damping effect because of the mass involved in the process is maximal in this case, hence the corresponding spectrum possesses the lowest median frequency $(1.65 \mathrm{~Hz})$.

The left diagram in the figure 2 also shows that the beginning of the $\theta$ deviation coincides with the peak of $\mathrm{P}$ wave of the ECG. P wave corresponds to the beginning of an intracardiac discharge of blood into the ventricles when cardiac muscle barely starts to contract, but blood is still almost motionless. This coincidence allows us to assume that the blood which is immobile (or moving steady) may rotate the radio wave polarization direction by a certain (zero or non-zero) constant angle (not changing in time). But the variations of the rotation angle could be associated only with blood acceleration or deceleration, i.e. a non stationary blood motion. Moreover, a non-zero PCG signal was recorded even after the cardiac contraction had finished. It was consistent with the reverse blood flow within the aorta and pulmonary artery, which closes the heart valves when a rapid bloodstream slowdown occurs.

Thus, we hypothesize that the polarization direction rotation is caused not only by heart displacement and rotation but rather by the moving blood properties.

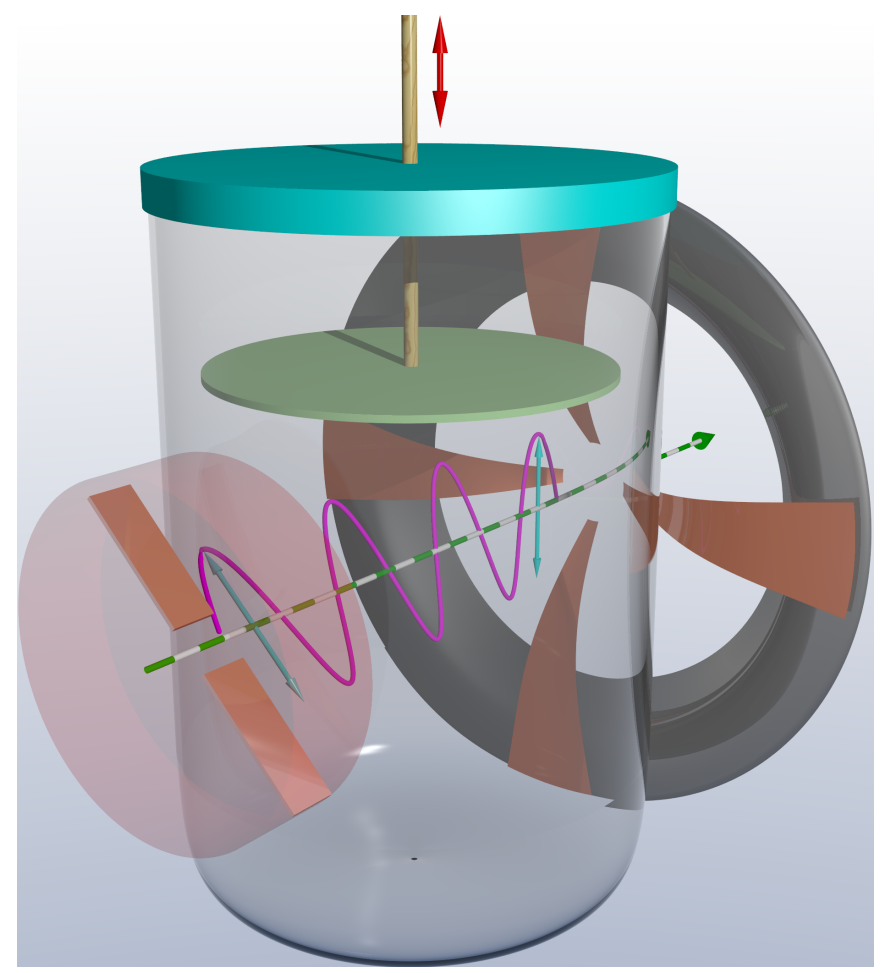

FIG. 3. The experimental setup scheme. A shortened transmitting antenna (surrounded on the sides by a radar absorbing case) is on the left while a receiving slot antenna with QAM is on the right. The relative orientation of the antennas polarization is $45^{\circ}$

\section{B. Reproducing the phenomenon in vitro}

\section{A simple setup}

In order to test experimentally our hypothesis about the dependence of polarization direction rotation on the blood movement as a conductive medium we assembled the setup illustrated by Figure 3 .

There is a covered container between the transmitting and receiving antennas. A thin polystyrene disk mounted on a long dielectric pusher is placed inside the container. The actuating pusher applies an external force to the disk and makes it move. The container is filled with an electrolyte solution without any air residues. Such setup implies no sway or shape change of the solution while the disk moving. Thereby the distortion of the scattering field affecting the measurements is observed or - simply put - mechanical excitation. The disk was displaced in axial direction by the pusher because of the weight change after the recording had started. The weight drop was provided either by a soft push through the shock-absorbing pad or by a sharp blow. The experiment has been carried out at ambient temperature of $+20^{\circ} \mathrm{C}$.

The absence of any response has been checked previously on the disk moving inside the empty container and one filled with distilled water. In the first case, there was no signal at all. In the second one, a barely noticeable signal was recorded at 
A new RF acoustic method

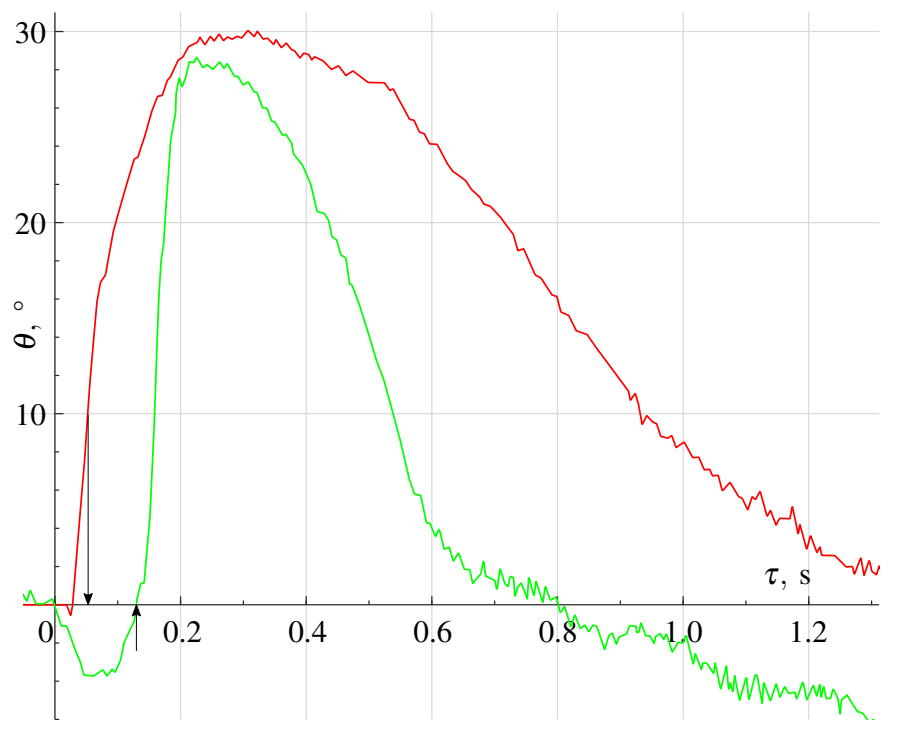

FIG. 4. The $\theta$ angle variation during a soft push of the disk (green curve). In the experiment on a tinted solution, it was seen that the jets flowing around the disk move in the opposite direction at a higher speed than the disk itself. A red curve means the same because of a sharp hit on the pusher, the jet flow differs from the previous observation. For both curves the external force starts to act at zero time and ends at time marked with arrow.

the noise level. The response signal disappeared completely when distilled water was replaced with bidistilled one.

Then we have held series of measurements on different solutions. First experiment has been carried out on distilled water and citric acid dissolving slowly within it. The experiment on distilled water and citric acid was the very first approximation of a cardiac system environment. Here we saw the response increased when the acid dissolved.

Further we have investigated the same process on $\mathrm{NaCl}$ and $\mathrm{KCl}$ solutions having approximately equal molar concentrations. The same equipment as for the cardiogram recording has been used.

It should be noted that these experiments are easy to implement and are highly repeatable. Despite this, the effect has remained unnoticed since the invention of radio communications.

\section{A mechanical excitation in simple setup}

When the container was filled with isotonic $\mathrm{NaCl}$ solution, any displacement of the disk always caused an appearance of a high-amplitude signal at the detector output. The amplitude of the output signal enhanced with the pusher acceleration increase. Figure 4 show the waveforms of the process. We did not find detectable differences in the response for $\mathrm{NaCl}$ and $\mathrm{KCl}$ solutions having approximately the same molar concentrations of $\approx 0.157 \mathrm{~mol} / \mathrm{l}$.

The experiment on more concentrated $\mathrm{NaCl}$ solution confirmed the growth of the response in accordance to the growth of concentration ceteris paribus. The response initially passed through the peak of intensity and decreased as the concen-

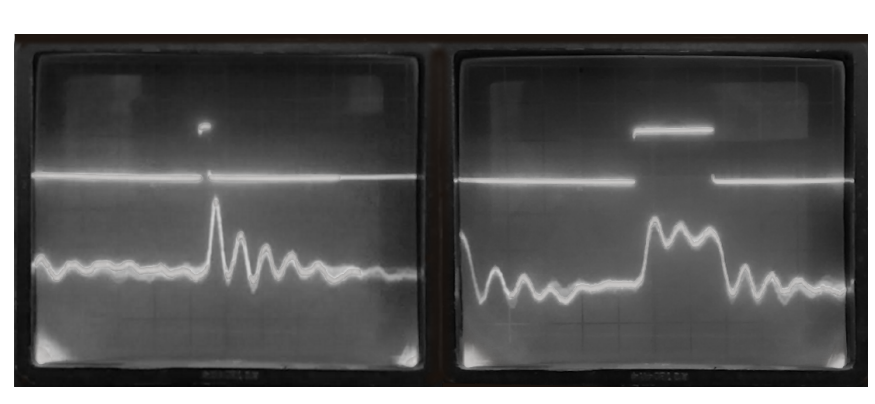

FIG. 5. Left waveforms show a system response on the repetitive acoustic pulse $1 \mathrm{~ms}$ long. Waveforms on the right correspond both to the pulse duration and repetition frequency increased. The damped oscillatory process is clearly visible, possibly due to an echo in the container. Upper beam shows a hydrophone input signal while the lower one is the op-amp output voltage. An electric power of the impulse applied to the hydrophone is equal to $1 \mathrm{~mJ}$. The polarization direction of the wave is deviated from the soundwave propagation vector by $45^{\circ}$. We were able to observe the effect confidently down to pulse energies of $1 \mu \mathrm{J}$
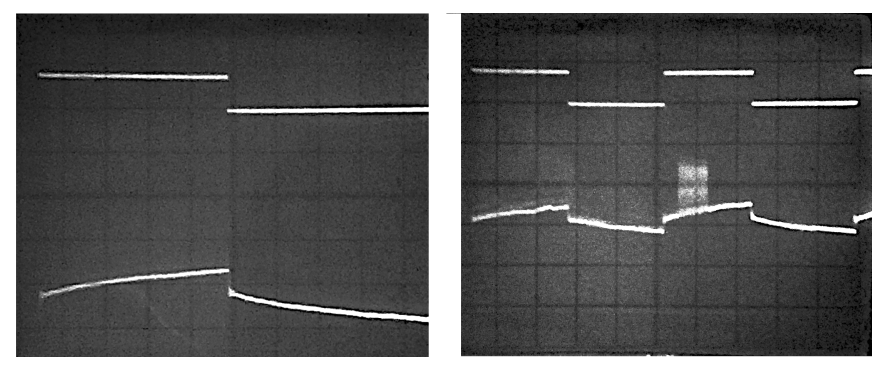

FIG. 6. A system response on the Heaviside function signal (upper beam) is shown on the left. Recording duration is $2.5 \mathrm{~s}$. The equipment bandwidth starts at zero frequency. It is easy to observe that after a rapid, momentary $\theta$ deviation because of the acoustic wave propagation, the further post-impulse rotation continues in the same direction. On the right is a $5 \mathrm{~s}$ fragment of the record that shows an unsuccessful attempt to interfere to post-impulse rotation process by turning on (briefly) an additional more powerful transmitter. Interference is visible on the lower beam of the oscilloscope

tration approached the saturation, and then the response was hardly observed within the saturated solution. We can make an assumption that the described effect is recorded by our equipment only for sufficiently concentrated but unsaturated solutions. We did not find detectable differences in the response for $\mathrm{NaCl}$ and $\mathrm{KCl}$ solutions having approximately the same molar concentrations. No experiments with radio frequency variation were carried out.

\section{An acoustical excitation in simple setup}

However, in order to find a supposed acoustic phenomenon we have held one more experiment under rather different conditionals. We recorded the response from the solution excited with an immersed into it hydrophone. The hydrophone 
was driven using MOSFET switch mode output stage and the whole equipment were tuned to zero frequency bandwidth (direct voltage). Figures 5 and 6 show the corresponding waveforms recorded from the operational amplifier output.

A completely unexpected behavior of the studied system has been revealed in the experiment with hydrophone. A reaction of electrolyte solution to the acoustic wave front passage was very prolonged contrary to our expectations. Thus, a long-term (in comparison with typical solvent relaxation times) "memory" was detected for the system.

As one may remember, we observed polarization direction rotation within the heart medium to be in conformity with ECG signal earlier in Section III A. But here we had a slow $\theta$ variation to be continued even after its rapid deviation at the moment of the acoustic wave passage. Thereby the anisotropy degree of the entire volume increased (see Fig. 6) within tens of seconds after the pulse. The $\theta$ angle value passed through the maximum and went then back to its initial value (before the pulse) after 30 seconds or even more.

This makes us conclude: when the wave front passes through the medium, anisotropy arises almost inertialessly, i.e. the times observed were less than $50 \mu$ s at the present installation (and they are most likely much less than this value). Anisotropy persists for at least tens of seconds after the wave front transit. It increases with time (see fig. 5, 6), then reaches its maximum, decreases and disappears. The passage of the acoustic wave reverse front changes the sign of the arisen anisotropy (see fig. 6).

In order to exclude the influence of RF energy "pumping" into the studied object we have attempted to interfere into the process with another transmitter which was more powerful $(+20 \mathrm{~dB})$ than the initially used one and had the same frequency (but different polarization direction). It was turned on for a very short time but had no effect on the process of post-impulse $\theta$ variation (see fig. 6). It is obvious that we can neglect the "observer effect", i.e. there is no changes in the structure of the observed object because of the radio signal passing through it.

Any manipulations with the power and/or modulation of the radio signal do not affect the time dependence of anisotropy (see fig. 6). Thus, a peculiar effect of "memory" is registered but it requires a consistent explanation. This way we proved a dependence of the magnitude of the resulting RF anisotropy on the energy of the front of the acoustic wave passing through the electrolyte solution, and its independence on the power of the RF transmitter.

Our experiments cannot yet provide a quantitative estimation of the phenomenon registered that is outwardly similar to the effects discovered by Kerr and Pokelson. It is not mentioned in the main physical reference books (for example, ${ }^{34}$ ). However, we have experimentally established now that the unsteady motion of electrolyte solution affects the polarization direction of the RF wave passing through it.

\section{DISCUSSION}

At the first consideration we assumed the mechanism of anisotropy occurence as next. When a liquid moves with alternating acceleration, the hydrodynamic forces drive cations and anions differently. It may occur due to non equal masses of ions, their different radii and hydration numbers. Hence the displacement of negative charges in relation to positive ones might arise.

In order to encourage further discussion first we have to mention that the difference of electric potential between two points of electrolyte solution, which is moving with alternating acceleration, was discovered earlier ${ }^{35}$ (english abstract ${ }^{36}$, remastered english version ${ }^{37}$ ). The mechanism of such potential appearing was considered as a shift of the opposite charges existing in plasma-like electrolyte, analogously to shift which causes the Langmuir waves in real plasma. However, the polarization direction rotation was not a matter this paper dealt with.

The opposite charges displacement, in turn, should cause the occurrence of parallel oriented water molecules statistic dipole structures in the solution. Then the polarization direction of radio signal passed through it rotates as the orthogonally polarized waves generated by the antenna have different speeds in an anisotropic medium. But our experiments showed the failure of such approach.

An intensity of response from $\mathrm{KCl}$ solution indicates the hydrodynamic explanation is at least insufficient. If our proposed hydrodynamic explanation were close to reality, then (ceteris paribus) the intensity would have been much less than one from $\mathrm{NaCl}$ solution because the dielectric friction coefficients for $\mathrm{K}^{+}$and $\mathrm{Cl}^{-}$in water are very close to each other at the range of temperatures from $+5^{\circ} \mathrm{C}$ up to $+25^{\circ} \mathrm{C}^{38[39}$. For the further development of the hydrodynamic model one may find usefull some novel theoretical approaches capable of the unsteady fluid flow description at the microscopic level, for example, the so-called theories of complex fluids ${ }^{40}$.

It is very likely that a simple hydrodynamic description of the observed effect, even within the framework of a more perfect model, cannot correctly explain the phenomenon of residual anisotropy, the so-called "memory". Normally it should not have occurred and an oscillogram was predicted to show an ordinary curve of a differentiating chain when the oscilloscope input is open.

Taking into account the thermal motion in the solution, one could hardly assume the presence of any molecular vibrational processes that cause such a long "memory" - they should have been leveled out by thermal noise rather quickly. Moreover, the total energy of the thermal motion of water molecules is immeasurably greater than the energy introduced into the system by an acoustic pulse. The energy of a pulse of $50 \mu$ s duration emitted by a $1 \mathrm{~W}$ powered hydrophone was estimated to be of the order of $50 \mu \mathrm{J}$. The front of such a wave, according to our data, rotates the polarization direction by $10^{\circ}$ approximately.

Let's assume the anisotropy to be caused precisely by the orientation of the water molecules dipole moments. Then the integral hodograph of the molecular motion, which is a sphere 
in an isotropic medium, should take a form of an ellipsoid with any significant difference of the major and minor semiaxes. One should take into account that the enthalpy of water at $+20^{\circ} \mathrm{C}$ is $84 \mathrm{~kJ} / \mathrm{kg}$ relatively to the triple point and the mass of the solution taken for our experiment was about $2 \mathrm{~kg}$. Thereby, it is hard to anticipate the acoustic pulse of the utilized hydrophone to be capable of introducing sufficient energy in accordance with our assumtion. The estimates of the energies of the acoustic pulse and the thermal motion of water differ by approximately 9 orders of magnitude.

Thus the most likely physical process that remains suitable for explaining the observed giant relaxation times is the interaction of the nuclear spin angular momentums of hydrogen atoms of water molecules 41 . And the closest to the observed response, in our opinion, is the process of spin glass magnetization shown at Figure 7 H2

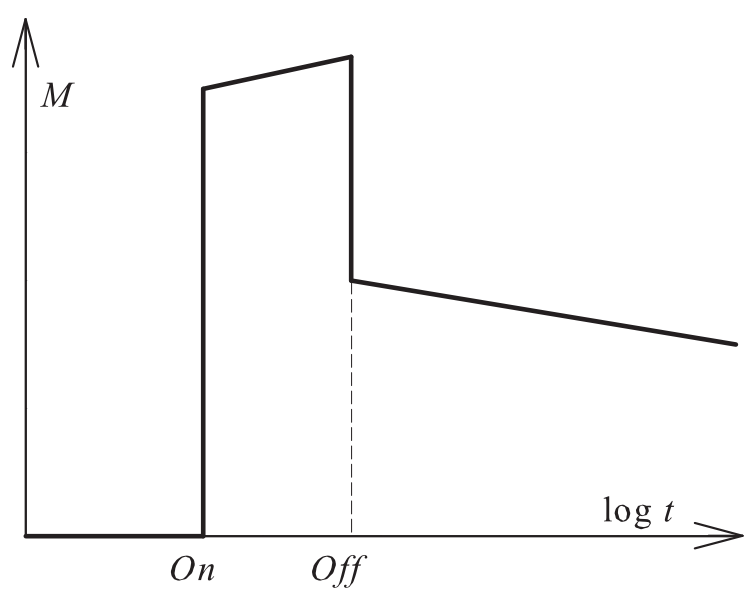

FIG. 7. Magnetization $M$ of spin glass as a function of time after turning on and off the magnetic field

One may use the properties of spin glass as a prototype and then refer the behaviour of the solution under the study to the Edwards-Anderson mode ${ }^{43}$. Id est, there is no random directions of spin, but a degeneracy in various spin configurations within the medium of an acoustic wave propagation. Withal the number of possible metastable states is infinitely large and they posses very long relaxation time. The authors cannot yet unambiguously correlate these states with the existing physical objects in the electrolyte solution (hydrated ions, individual water molecules, or their clusters) to unravel the problem.

Of course, an exhaustive theoretical justification is required here. Also, some hope is given by the known experimental fact that two spin isomers of water can be separated under very specific conditions 44 . In this case the time that equilibrium between water isomers takes to establish, coincides (in order of magnitude) with the relaxation times we observe.

\section{v. CONCLUSIONS}

Summarizing our experiment we can highlight the following.
1. Acoustic or mechanical excitation of pure water does not cause any discernible polarization direction rotation. Keeping the constant level of acoustic excitation we have revealed that the $\theta$ rotation angle grows with increasing concentration of electrolyte in water. The response decreased when $\mathrm{NaCl}$ solution started to slightly approach the saturation and for the saturated solution it practically ceased on our equipment.

2. An experiment with $\mathrm{KCl}$ solution having a molar concentration of $0.157 \mathrm{~mol} / \mathrm{l}$, which is close to that for an isotonic $\mathrm{NaCl}$ solution, did not reveal an experimentally detectable difference in response between them.

3. The magnitude of the resulting RF anisotropy depends on the energy of the front of the acoustic wave passing through the electrolyte solution and does not depend on the power of the RF transmitter.

4. When the wave front passes through, anisotropy arises almost inertialessly, i.e. the times observed were less than $50 \mu \mathrm{s}$ at the existing installation (and they are most likely much less than this value).

5. Anisotropy persists for at least tens of seconds after the wave front passing. It even increases with time (see fig. 5, 6), then reaches a maximum, decreases and dissapears. This turned out to be completely unexpected. Any manipulations with the power and/or modulation of the radio signal do not affect the time dependence of anisotropy (see fig. 6). Thus, a peculiar effect of "memory" is registered, which requires a consistent explanation.

6. The passage of the reverse front of the acoustic wave changes the sign of the anisotropy arisen (see fig. 6).

And, finally, we would like to share our view on the prospects of the observed phenomenon.

1. A completely non-contact method may be promising for industrial applications, e.g. for monitoring liquids moving through the pipelines, detecting unacceptable vibrations, turbulences etc. We suppose the method to be applicable for fundamental physics research, as well as for various branches of industry, biology and medicine, oceanography and geology.

2. The sensitivity and selectivity of sonar stations may be increased due to the use of a polarimetric sensor as a hydrophone. Currently, the phase transition of sound waves passing through the propagation medium to a sensitive element, as in all existing microphones, is eliminated.

3. Measurement of the angle of RF polarization direction after the passage of a single short acoustic pulse through the body tissues allows us to develop a fundamentally new method of single-pulse ultrasound introscopy. 
4. In our opinion, the effect can be widely used in biophysics an medicine, taking into consideration the nessesity for non-contact research methods. Monitoring of operator's vital statistics is the case, e.g. the car driver's heart rate and integral assessment of the heart pumping function.

5. The practical application of the effect detected is already possible using the developed equipment and signal processing algorithms, since the proposed sensor will perform the functions of a "finite state machine" (i.e. time-invariant transcoding). The reference data in read-only memory is enough for it to work.

6. We believe that the theoretical substantiation of the observed phenomenon requires wide cooperation of specialists in various fields.

\section{ACKNOWLEDGMENTS}

Our gratitude to prof. Alexander G. Nerukh, the head of the Department of Higher Mathematics of Kharkov National University of Radio Electronics, to Igor I. Melnik, the associate professor of the Department of Organic Chemistry and Biotechnology of the National Technical University "Kharkov Polytechnic Institute", to Andrey N. Nechay, the researcher at the Institute of Microstructure Physics of the Russian Academy of Sciences for an efficient discussion of the work results and conclusions.

${ }^{1}$ M. I. Finkel'shteyn, V. G. Gloushnev, and A. N. Petrov, On the anisotropy of the radiowave attenuation in the sea ice International Meeting on Radioglaciology, Lyngby, May 1970. Proceedings (1970) p. 159.

${ }^{2}$ E. I. Kaleri, A. M. Kluga, A. N. Petrov, and M. I. Finkel'shteyn, "Anisotropy of radiowave delay in sea ice," Akademiia nauk SSSR. Izvestiia. Atmospheric and oceanic physics (1971)

${ }^{5}$ V. V. Bogorodskiy and G. P. Khokhlov, "Anisotropy of the microwave dielectric constant and absorption coefficient of arctic drift ice," Soviet physics. Technical physics (1977)

${ }^{4}$ K. Matsuoka, T. Furukawa, S. Fujita, H. Maeno, S. Uratsuka, R. Naruse, and O. Watanabe, "Crystal orientation fabrics within the antarctic ice sheet revealed by a multipolarization plane and dual-frequency radar survey," Journal of Geophysical Research: Solid Earth 108 (2003), 10.1029/2003JB002425 https://agupubs.onlinelibrary.wiley.com/doi/pdf/10.1029/2003JB002425

${ }^{5}$ C. Wang, Z. Dong, X. Zhang, X. Liu, and G. Fang, "Method for anisotropic crystal-orientation fabrics detection using radio-wave depolarization in radar sounding of mars polar layered deposits," IEEE Transactions on Geoscience and Remote Sensing 56, 5198-5206 (2018)

${ }^{6} \mathrm{~S}$. Missaoui and M. Kaddour, "A novel combined-design of an antennafilter rf with optical anisotropy of nematic liquid crystal for uwb applications," OPTOELECTRONICS AND ADVANCED MATERIALS-RAPID COMMUNICATIONS 13, 209-216 (2019)

${ }^{7}$ R. J. Prance, A. Debray, T. D. Clark, H. Prance, M. Nock, C. J. Harland, and A. J. Clippingdale, "An ultra-low-noise electrical-potential probe for human-body scanning," Measurement Science and Technology 11, 291297 (2000)

${ }^{8}$ C. Harland, T. Clark, and R. Prance, "Electric potential probes-new directions in the remote sensing of the human body," Measurement Science and Technology 13, 163 (2001)

${ }^{9}$ E. Pinheiro, O. Postolache, and P. Girão, "Contactless impedance cardiography using embedded sensors," Measurement Science Review 9, 157-164 (2013)
${ }^{10}$ D. B. Jain, J. Weeks, D. Nadezhdin, and J.-F. Asselin, “Contactless electric cardiogram system," (2016).

${ }^{11}$ V. A. Simon, Razrabotka i issledovanie sredstv registracii $i$ obrabotki biosignalov dlja upravlenija rezhimami raboty medicinskih kompleksov, Ph.D. thesis Federal'noe gosudarstvennoe avtonomnoe obrazovatel'noe uchrezhdenie vysshego obrazovanija «Sankt-Peterburgskij gosudarstvennyj jelektrotehnicheskij universitet «LJeTI» im. V. I. Ul'janova (Lenina)» (2019).

${ }^{12}$ A. Tariq, Vital Signs Monitoring Using Doppler Radar and On-Body Antennas, Ph.D. thesis. School of electronic, electrical and computer engineeringcollege of engineering and physical science, University of Birmingham (2012).

${ }^{13}$ M. Pour Ebrahim, M. Sarvi, and M. R. Yuce, "A doppler radar system for sensing physiological parameters in walking and standing positions," Sensors (Basel) 17, 485 (2017)

${ }^{14} \mathrm{D}$. Buxi, J. Redouté, and M. R. Yuce, "Blood pressure estimation using pulse transit time from bioimpedance and continuous wave radar," IEEE Transactions on Biomedical Engineering 64, 917-927 (2017)

${ }^{15}$ D. Buxi, E. Hermeling, M. Mercuri, F. Beutel, R. G. van der Westen, T. Torfs, J. Redouté, and M. R. Yuce, "Systolic time interval estimation using continuous wave radar with on-body antennas," IEEE Journal of Biomedical and Health Informatics 22, 129-139 (2018)

${ }^{16}$ F. Michler, K. Shi, S. Schellenberger, T. Steigleder, A. Malessa, L. Hameyer, N. Neumann, F. Lurz, C. Ostgathe, R. Weigel, and A. Koelpin, "A clinically evaluated interferometric continuous-wave radar system for the contactless measurement of human vital parameters," Sensors 19, 2492 (2019)

${ }^{17} \mathrm{C}$. Wolff, "The radar range equation," (1998)

${ }^{18}$ SanPiN 2.2.4/2.1.8.055-96 - Jelektromagnitnye izluchenija radiochastotnogo diapazona (JeMI RCh). Sanitarnye pravila i normy (2002).

1947 CFR \$ 2.1093 - Radiofrequency radiation exposure evaluation: portable devices.

${ }^{20}$ BS EN 62209-2:2010 - Human exposure to radio frequency fields from hand-held and body-mounted wireless communication devices - Human models, instrumentation, and procedures - Part 2: Procedure to determine the specific absorption rate (SAR) for wireless communication devices used in close proximity to the human body (frequency range of $30 \mathrm{MHz}$ to $6 \mathrm{GHz}$ ) (2010).

${ }^{21} \mathrm{H}$. McPherson, "An x-band frequency agile source with extremely low phase noise for doppler radar," in 92 International Conference on Radar (1992) pp. 292-295.

${ }^{22} \mathrm{~S}$. Farahani, "Chapter 5 - rf propagation, antennas, and regulatory requirements," in ZigBee Wireless Networks and Transceivers. edited by S. Farahani (Newnes, Burlington, 2008) pp. $171-206$.

${ }^{23}$ A. A. Shlykov and A. K. Saraev, "Osobennosti jelektromagnitnogo polja gorizontal'nogo jelektricheskogo dipolja v metode radiomagnitotelluricheskih zondirovanij s kontroliruemym istochnikom," Vestnik SPbGU. Nauki o Zemle (2013)

${ }^{24} \mathrm{~A}$. Hayat and M. Faryad, "On the radiation from a hertzian dipole of a finite length in the uniaxial dielectric medium," OSA Continuum 2, 1411-1429 (2019)

${ }^{25}$ Z. J. Juzbashev, J. I. Skvorcov, and T. M. Bogdanova, "Apikal'naja sejsmokardiografija po b.s.bozhenko kak instrument issledovanija transmitral'nogo diastolicheskogo krovotoka i ocenki funkcii rasslablenija miokarda," Sovremennye problemy nauki i obrazovanija (2015)

${ }^{26} \mathrm{~V}$. N. Tatarinov, S. V. Tatarinov, and L. P. Ligthart, Vvedenie v sovremennuju teoriju poljarizacii radiolokacionnyh signalov. Poljarizacija ploskih jelektromagnitnyh voln i ee preobrazovanija edited by A. I. Kozlov, Polarizacija radiovoln, Vol. 2 (Izdatelstvo Tomskogo universiteta, 2006).

27 "The video of contactless radar electrocardiography working," (2019).

${ }^{28} \mathrm{~F}$. J. Harris, "On the use of windows for harmonic analysis with the discrete fourier transform," Proceedings of the IEEE 66, 51-83 (1978).

${ }^{29}$ V. M. Shipulin, S. L. Andreev, J. J. Vecherskij, and E. P. Gordov, Ispol'zovanie lazerov $v$ serdechno-sosudistoj hirurgii: ot jeksperimenta $k$ praktike (STT, 2010).

${ }^{30} \mathrm{~V}$. M. A. Aed, Razvitie metodov i algoritmov obrabotki i nejrosetevogoanaliza fonokardiosignala, Ph.D. thesis Federal'noe gosudarstvennoe bjudzhetnoe obrazovatel'noe uchrezhdenievysshego obrazovanija «Vladimirskij gosudarstvennyj universitetimeni Aleksandra Grigor'evicha i Nikolaja Grigor'evicha Stoletovyh» (V1GU) (2017). 
${ }^{31}$ Y. Gorshkov, "Visualization of the heart sounds," Scientific Visualization 9 , 97-111 (2017)

${ }^{32}$ A. A. Kedrov and T. J. Liberman, "O tak nazyvaemoj reokardiografii," Klinicheskaja medicina 27, 40-46 (1949).

${ }^{33}$ J. nyboer, M. M. Kreider, and L. Hannapel, "Electrical impedance plethysmography," Circulation 2, 811-821 (1950)

${ }^{34}$ J. Rumble, ed., CRC Handbook of Chemistry and Physics, 100th Edition (CRC Press, 2019).

${ }^{35}$ A. V. Kramarenko, G. P. Kulemin, and A. M. Savchuk, "Gemodinamicheskij komponent jelektrokardiogrammy," Biofizika 41, 686-694 (1996)

${ }^{36}$ A. V. Kramarenko, G. P. Kulemin, and A. M. Savchuk, "The hemodynamic component of an electrocardiogram," (1996).

${ }^{3 /}$ A. V. Kramarenko, G. P. Kulemin, and A. M. Savchuk, "Hemodynamic contribution to human ecg," (1996).

${ }^{38}$ M. Ueno, A. Yoneda, N. Tsuchihashi, and K. Shimizu, "Solvent isotope effect on mobilities of potassium and chloride ions in water at high pressure. ii. a low temperature study," The Journal of Chemical Physics 86, 4678-
4683 (1987)

${ }^{39}$ M. Ueno, N. Tsuchihashi, and K. Shimizu, "Solvent isotope effect on mobilities of potassium and chloride ions in water at high pressure. iii. a high temperature study," The Journal of Chemical Physics 92, 2548-2552 (1990)

${ }^{40}$ B. Eisenberg, "Mass action in ionic solutions," Chemical Physics Letters 511, 1-6 (2011)

${ }^{41}$ J. Casanueva, J. S. Fabiàn, E. Dièz, and A. Esteban, "Nmr spin-spin coupling constants in water molecule: equilibrium and rovibrational values," Journal of Molecular Structure 565-566, 449 - 454 (2001)

${ }^{42}$ V. Kincel', "Spinovye stekla kak model'nye sistemy dlja nejronnyh setej," Usp. fiz. nauk 152, 123-131 (1987)

${ }^{43}$ S. F. Edwards and P. W. Anderson, "Theory of spin glasses," Journal of Physics F: Metal Physics 5, 965-974 (1975)

${ }^{44}$ V. I. Tikhonov and A. A. Volkov, "Separation of water into its ortho and para isomers," Science 296, 2363-2363 (2002) 